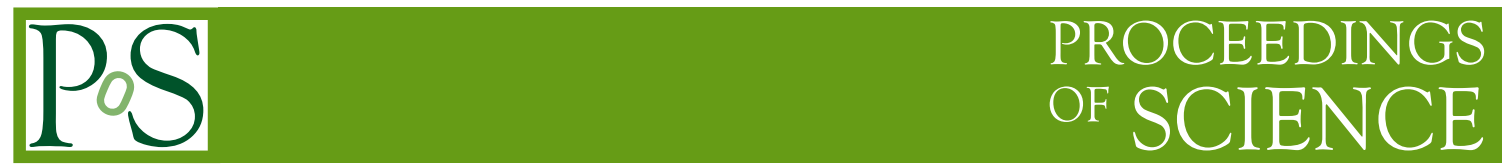

\title{
Recent H1 Results on Heavy Flavour Photoproduction
}

\section{Michel SAUTER ${ }^{* \dagger}$}

University Heidelberg, Physikalisches Institut

E-mail: michel.sauter@desy.de

The production of charm and beauty quarks in $e p$-scattering at HERA has been studied by the H1 collaboration over a wide phase space range. Data from charm and beauty photoproduction $\left(Q^{2} \approx 0 \mathrm{GeV}^{2}\right)$ are a good test for perturbative Quantum Chromodynamics. The measured heavy flavour cross sections are reasonably well described by next-to-leading order calculations.

The European Physical Society Conference on High Energy Physics -EPS-HEP2013

18-24 July 2013

Stockholm, Sweden

* Speaker.

On behalf of the H1 collaboration. 


\section{Introduction}

The various measurements of charm and beauty production at the electron-proton collider HERA, based on different experimental techniques and covering a wide phase space range, are important to test the accuracy of perturbative Quantum Chromodynamics (pQCD). In leading order (LO) QCD, the dominant contribution to heavy flavour production in $e p$-collisions, $e p \rightarrow e c \bar{c} X$, $e b \bar{b} X$, is the photon-gluon fusion (PGF) process, in which a photon emitted from the electron fuses with a gluon from the proton to a charm- or beauty-quark pair as depicted in figure 1 a. Resolved processes where the photon fluctuates into a hadronic state before undergoing a hard collision, as indicated in figure 10 b contribute to a lower extent. Predictions derived in the framework of pQCD are expected to be reliable whenever an experimental hard scale, $\mu$, is available, with $\mu$ being much larger than $\Lambda_{Q C D}$. Since the charm- and beauty-quark masses are larger than $\Lambda_{Q C D}$, this condition is fulfilled for charm or beauty production. Depending on the measurement, two additional experimental hard scales may be available: the square root of the photon virtuality, $Q^{2}$, and the transverse energy or momentum of the heavy-quark.

Three analyses recently released by the $\mathrm{H} 1$ collaboration are discussed, measuring heavy flavour production in photoproduction, where the exchanged photon is almost real $\left(Q^{2} \approx 0 \mathrm{GeV}^{2}\right)$ and does not provide a hard scale.

a)

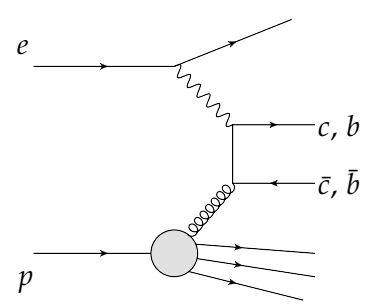

b)

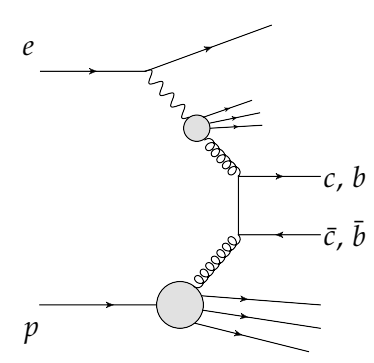

Figure 1: Generic leading order diagrams for $c \bar{c}$ or $b \bar{b}$ production in $e p$ collisions. The diagram a) is referred to as direct or pointlike, the diagram $b$ ) is referred to as resolved or hadronlike.

\section{Theoretical aspects}

Perturbative calculations of heavy-quark production at HERA are available up to next-toleading order (NLO) in $\alpha_{s}$. A number of theoretical approaches exist to describe heavy flavour quark production in $e p$-collisions. The different theoretical approximations of heavy flavour quark production are formulated in different theoretical schemes, treating the heavy quark either as massive and produced dynamically (massive scheme), or as massless and as an active constituent of the proton at large scales (massless scheme). The massive scheme is expected to describe the data better at experimental scales of the same order as the heavy quark mass. For predictions in the massive scheme the programs FMNR [1] and MC@NLO [目] are used. The massless scheme is expected to be valid in regions of the phase space involving larger experimental scales. The general mass variable flavour number scheme (GM VFNS) [3] for heavy quarks interpolates from the massive approach at low scales to the massless approach at high scales. 


\section{Experimental aspects}

\subsection{The H1 Detector at HERA}

The ring collider HERA accelerated electrons and protons to energies of $E_{e} \simeq 27.6 \mathrm{GeV}$ and $E_{p} \simeq 920 \mathrm{GeV}$. Inside the two experiments $\mathrm{H} 1$ and ZEUS the two beams were brought to collision, resulting at a centre of mass energy of $\sqrt{s} \simeq 320 \mathrm{GeV}$. HERA was operated from 1992-2007 in two operation periods and delivered to each experiment a data set corresponding to an integrated luminosity of $L \simeq 0.5 \mathrm{fb}^{-1}$. H1 was a classical, asymmetric multi-purpose detector designed for the measurement of electron-proton interactions, a detailed description can be found in $\llbracket$.

\subsection{Experimental heavy flavour tagging methods at HERA}

Due to the heavy quark masses charm and beauty photoproduction at HERA are suppressed with respect to photoproduction of light flavoured quarks $(u, d, s)$. The corresponding cross sections and consequently event rates very roughly behave like: $\sigma_{u d s}: \sigma_{c}: \sigma_{b} \approx 2000: 50: 1$. For charm a full reconstruction of the $D$-mesons is possible. This is done preferentially using so-called $D^{*}$-tags, where the $D^{*}$-meson decays to $D^{0} \pi$ and the reconstruction of subsequent $D^{0}$ decays to $K \pi$ are exploited. For beauty, however, no suitable decay channel exists which provide enough statistics when being fully reconstructed. Therefore other tagging strategies are applied, which are based on the large quark masses, the long lifetime and the large lepton-decay branching ratio, $b \rightarrow \ell+X$, of $B$-hadrons. In figure 2 distributions are shown, relevant for the different heavy flavour tagging methods used in the analyses presented here.

\section{Recent measurements}

The H1 collaboration recently released a measurement of charm photoproduction [5] based on the reconstruction of $D^{*}$-mesons already during the data record [6]. The offline analysis yields to differential cross sections of high precision, which are presented as function of several kinematic variables. The measurement is compared to NLO pQCD predictions calculated in the massive scheme and the GM VFNS. Generally the predictions show, within their rather large uncertainties, reasonable agreement with the data. In figure 3 a) the single differential cross section for $D^{*}$ production is shown as a function of the pseudo rapidity of the $D^{*}$ meson. The analysis is extended by requiring two jets in addition to the tagged $D^{*}$-meson. These additional jets introduce an additional hard scale applicable for $\mathrm{pQCD}$ convergence. Two variables are of particular interest when studying charm di-jet production: $x_{\gamma}$ which corresponds in leading order QCD to the photon momentum carried by the jets and the azimuthal difference between the two jets $\Delta \varphi$. The variable $x_{\gamma}$ can discriminate, interactions with a pointlike photon (direct processes) from processes where the photon has a resolved hadronic structure. For the observable $\Delta \varphi$ a back-to-back topology is expected in LO QCD, while radiation of additional gluons leads to significantly smaller opening angles. Differential cross sections as function of $\Delta \varphi$ are therefore a good test for NLO calculations. In figure $3 \mathrm{~b}$ ) the cross section differential in $\Delta \phi$ is compared to the predictions of MC@NLO for the resolved enhanced phase space. In this region the NLO calculation is significantly below the data.

The $\mathrm{H} 1$ collaborations has also published a simultaneous measurement of charm and beauty jets in 
photoproduction [7] with various differential cross sections. The analysis requires a muon and two jets. The heavy flavour contribution to the data set is determined from fits to the distribution of the impact parameter $\delta$ of the muon track and the relative transverse momentum $p_{\mathrm{T}}^{\text {rel }}$ of the muon with respect to the nearest jet axis (figure $2 \mathrm{~b}$ ). As an example in figure $\bigoplus$ the charm and beauty jet cross sections as a function of the transverse momentum of the jet are shown. The measurement agrees reasonably well with the NLO pQCD predictions as calculated with the program MC@NLO.

In another measurement the [8] $\mathrm{H} 1$ collaboration has measured beauty photoproduction near threshold, by reconstructing beauty quarks at lowest transverse momenta, i.e. in a phase space region where the only available hard scale is the mass of the beauty quark. This is achieved by using the decay channel $b \bar{b} \rightarrow e e X$. Low $p_{\mathrm{T}}$ electrons were identified on trigger level [6, 9], and offline via a dedicated electron reconstruction algorithm having an excellent efficiency and background rejection behaviour. The beauty signal is discriminated against background by exploiting di-electron correlations based on the invariant di-electron mass, the azimuthal angle between the electrons, and product of the electron charges (figure $1 \mathrm{~g}$ ). The unfolded differential cross section as function of the mean transverse momentum of the beauty-quark pair is compared to the massive NLO-QCD prediction from FMNR in figure 5. The figure also shows the ratio of the measured cross section and the NLO QCD cross section. The uncertainties of the measurement are smallest at low $\left\langle P_{T}(b)\right\rangle$, where the cross section is largest. The theoretical prediction of the differential cross section agrees with the measurement within the large experimental and theoretical uncertainties.

\section{Conclusion}

Heavy flavour photoproduction at HERA is intensively studied in many analyses with various heavy flavour tagging methods, achieving high experimental precision. The heavy flavour production measurements are able to test pQCD at different scales. In general the discussed pQCD calculations describe the data reasonably well in shape and normalization.

\section{References}

[1] S. Frixione, M. L. Mangano, P. Nason and G. Ridolfi, Adv. Ser. Direct. High Energy Phys. 15 (1998) 609 [hep-ph/9702287].

S. Frixione, P. Nason and G. Ridolfi, Nucl. Phys. B 454 (1995) 3, [hep-ph/9506226].

S. Frixione, M. L. Mangano, P. Nason and G. Ridolfi, Phys. Lett. B 348 (1995) 633, [hep-ph/9412348].

[2] S. Frixione and B.R. Webber, JHEP 0206 (2002) 029 [hep-ph/0204244];

S. Frixione, P. Nason and B.R. Webber, JHEP 0308 (2003) 007 [hep-ph/0305252].

T. Toll and S. Frixione, Phys. Lett. B703 (2011) 452 [arXiv:1106.1614].

[3] G. Kramer and H. Spiesberger, Eur. Phys. J. 38 (2004) 309 [hep-ph/0311062].

B.A. Kniehl, G. Kramer, I. Schienbein and H. Spiesberger, Eur. Phys. J. 62 (2009) 365 [arXiv:0902.3166].

[4] I. Abt et al. [H1 Collaboration], Nucl. Instrum. Meth. A 386 (1997) 310;

I. Abt et al. [H1 Collaboration], Nucl. Instrum. Meth. A 386 (1997) 348. 
[5] F. D. Aaron et al. [H1 Collaboration], Eur. Phys. J. C 72 (2012) 1995 [arXiv:1203.1170].

[6] A. W. Jung et al., Proc. 15th IEEE-NPSS Real-Time Conference, (2007) 1.

[7] F. D. Aaron et al. [H1 Collaboration], Eur. Phys. J. C 72 (2012) 2047 [arXiv:1205.2495].

[8] F. D. Aaron et al. [H1 Collaboration], Eur. Phys. J. C 72 (2012) 2148 [arXiv:1206.4346].

[9] B. Olivier et al. Nucl. Instrum. Meth. A 641 (2011) 58 (Corrigendum).

a)

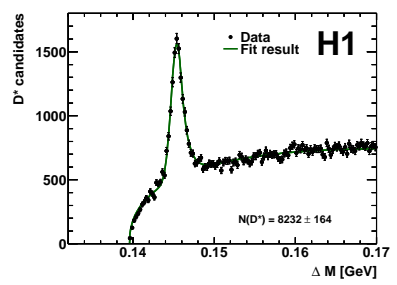

b)

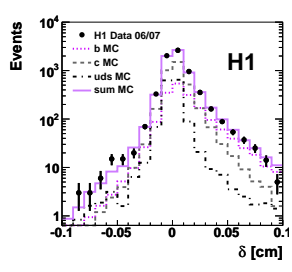

c)
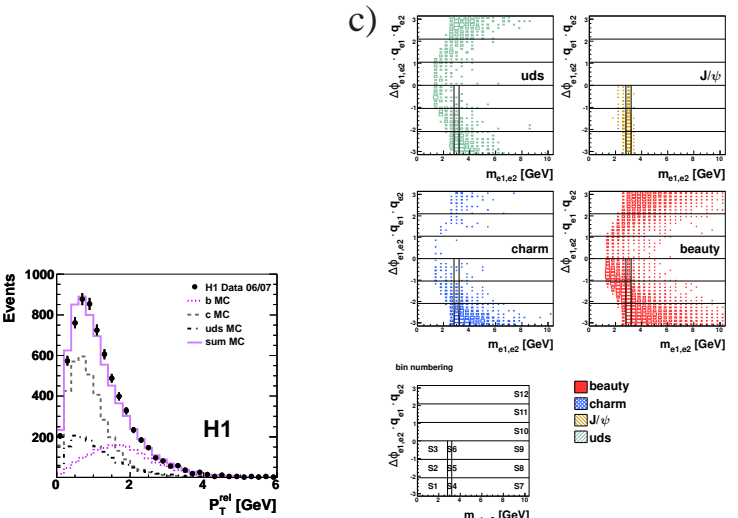

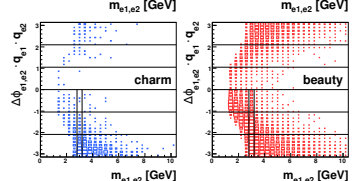

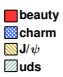

Figure 2: Heavy flavour tagging methods used: a) $D^{*}$ peak of [5] from fully reconstructed $D^{*}$ mesons, b) impact parameter $\delta$ and $P_{T}^{\text {rel }}$ distributions of [7] exploiting mass and lifetime information, c) templates of [8] based on di-electron correlations, in the variables $\Delta \phi_{e 1, e 2} \cdot q_{e 1} \cdot q_{e 2}$ defined by the charges multiplied with the azimuthal angle difference of the two electron candidates and the invariant mass $m_{e 1, e 2}$ of the two electron candidates, for light quarks ( $u d s), J / \psi, c$ and $b$ obtained by MC simulations.

a)

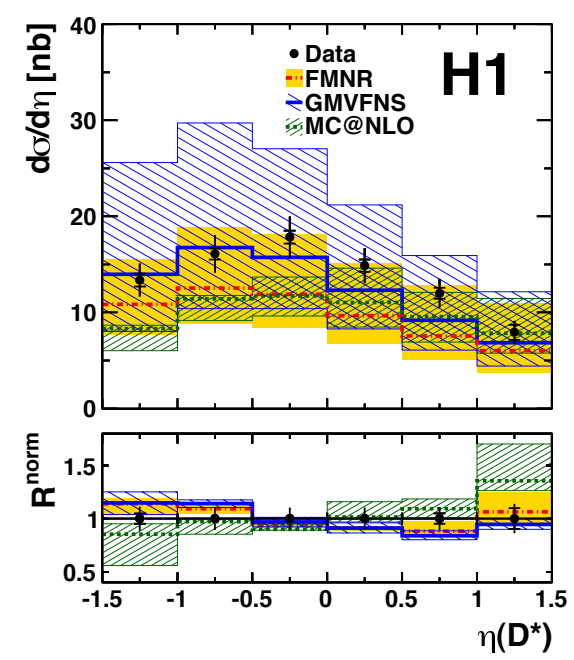

b)

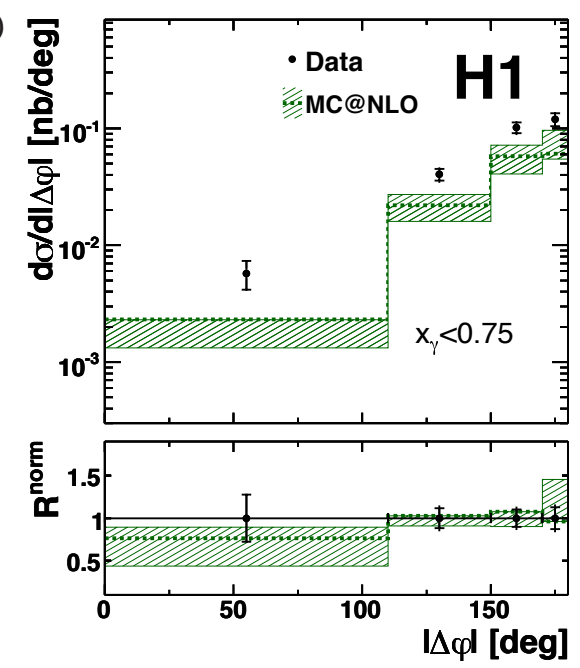

Figure 3: Left: Single differential $D^{*}$ cross section of analysis [ [\$] as a function of $\eta\left(D^{*}\right)$ compared to the NLO predictions of FMNR, GM VFNS and MC@NLO. Right: Single differential cross section for $D^{*}$-tagged dijet production as a function of the difference in azimuthal angle $\Delta \varphi$ compared to MC@NLO predictions. The data are shown as points with the inner errors being the statistical errors and the outer errors the total uncertainty. The bands indicate the NLO predictions including the theoretical uncertainty. The normalised ratio $\mathrm{R}^{\text {norm }}$ is shown as well. 
a)

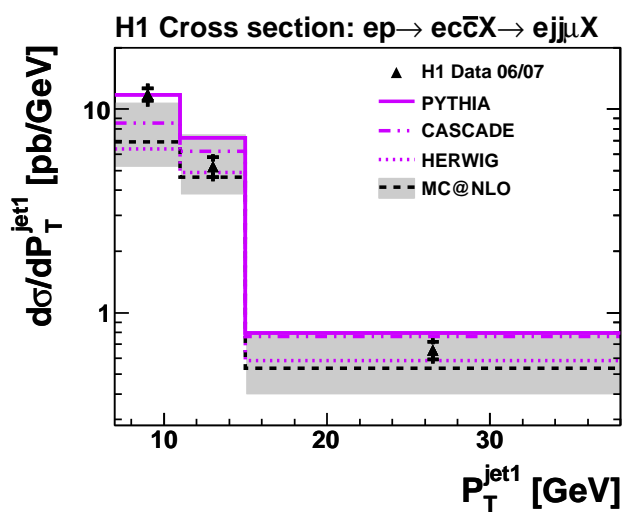

b)

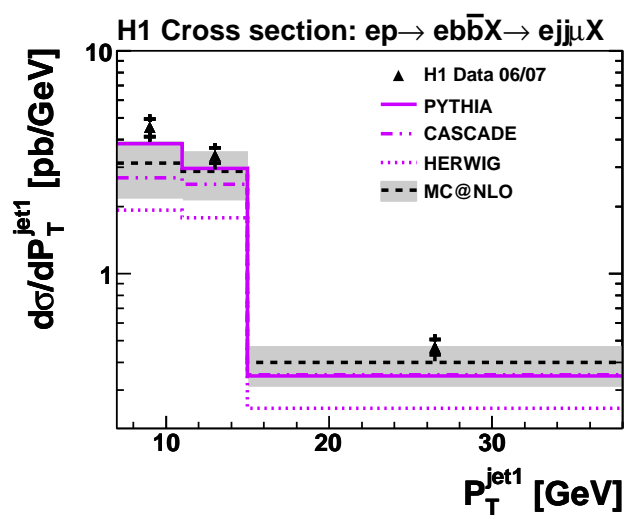

Figure 4: Differential cross sections for charm and beauty photoproduction of dijet events with a muon as a function of the transverse jet momentum, [7]. The inner error bars show the statistical errors, the outer error bars represent the statistical and systematic errors added in quadrature. The data are compared with the predictions from various LO models as well as to the NLO predictions of MC@NLO. The theoretical uncertainties of MC@NLO are given as shaded band.

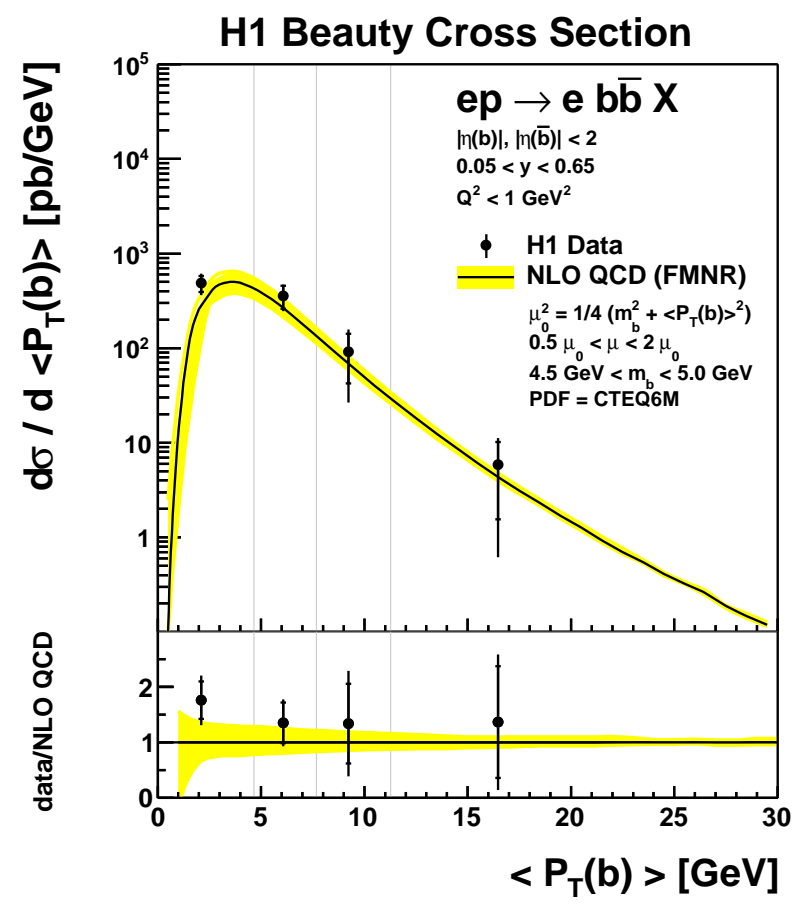

Figure 5: Upper part: Differential beauty cross section $\mathrm{d} \sigma / \mathrm{d}\left\langle P_{T}(b)\right\rangle$ as function of the quadratically averaged transverse momentum of the beauty quarks $\left\langle P_{T}(b)\right\rangle$, [8]. The data are represented by points with inner vertical error bars representing the statistical errors and outer error bars representing the total error. The vertical gray lines indicate the bin boundaries in $\left\langle P_{T}(b)\right\rangle$ of each data point and the points are shown at the bin centred positions. The data are compared to the FMNR NLO QCD calculation (solid line) with the uncertainty represented as shaded band. Lower part: Shown is the ratio of the measured cross section to the calculated NLO QCD prediction, $R=\frac{\mathrm{d} \sigma_{\text {measured }}}{\mathrm{d}\left\langle P_{T}(b)\right\rangle} / \frac{\mathrm{d} \sigma_{\mathrm{NLO}} \mathrm{QCD}}{\mathrm{d}\left\langle P_{T}(b)\right\rangle}$. 